\begin{tabular}{|c|l|}
\hline Title & Metal Induced Gap States at tetratetracontane/Cu Interface \\
\hline Author(s) & Kiguchi, M.; Y oshikawa, G.; Saiki, K.; A rita, R.; A oki, H. \\
\hline Citation & $\begin{array}{l}\text { Journal de Physique IV, 132, 199-203 } \\
\text { https://doi.org/L0.1051jp4:2006132038 }\end{array}$ \\
\hline Issue Date & 2006-03 \\
\hline Doc URL & http://hdl.handle.net/2115/8504 \\
\hline Rights & ○ EDP Sciences 2006 \\
\hline Type & article (author version) \\
\hline File Information & kiguti-v1.pdf \\
\hline
\end{tabular}

Instructions for use 


\title{
Metal Induced Gap States at tetratetracontane/Cu Interface
}

\author{
M. Kiguchi' ${ }^{1}$ G. Yoshikawa², K. Saiki², R. Arita ${ }^{3}$, H. Aoki ${ }^{3}$ \\ ${ }^{1}$ Div. Chemistry, Graduate School of Science, Hokkaido Univ., Sapporo, Hokkaido, Japan \\ 2 Dep. Compl. Sci. \& Eng., Grad. Sch. Frontier Sci., Univ. Tokyo, Kashiwa, Chiba, Japan \\ ${ }^{3}$ Dep. Physics, Univ. Tokyo, Hongo, Tokyo 113-0033, Japan
}

\begin{abstract}
We have shown, both experimentally and theoretically, that the metal-induced gap states (MIGS) can exist in epitaxially grown organic-insulator/metal interfaces. The material chosen is tetratetracontane $/ \mathrm{Cu}(001)$ with a well-defined interface. The element-selective near edge $\mathrm{x}$-ray absorption fine structure (NEXAFS) exhibits a pre-peak indicative of MIGS. An ab-initio electronic structure calculation supports the existence of the MIGS, and shows how the MIGS wave function looks like.
\end{abstract}

\section{INTRODUCTION}

It is well known that the characteristics of devices such as organic electroluminescence or organic solar cells crucially depend on the energy-level alignment at the organic/metal interface. The band alignment, as measured with photoemission spectroscopy and Kelvin probe, has been discussed for explaining various effects. However, very little is known about the band alignment at organic / metal interfaces. For inorganic-semiconductor/metal interfaces there is a mounting body of evidence that the band alignment is determined by the interface states such as defects states or metal-induced gap states. So the study of interface states at organic/metal interfaces should be imperative. Specifically, the interface states at organic-insulator/metal interfaces are intriguing, since their band alignment cannot be explained by simply assuming a common vacuum level. So the existence of the interface states is suggested, while the simple band picture dictates that the states are irrelevant for insulator/metal interfaces. So we look into this problem by combining experimental and theoretical studies.

\section{EXPERIMENTAL}

The experiments were performed with a UHV chamber at the soft x-ray beam line BL-7A at the Photon Factory, Tsukuba. Material chosen here is a typical organic insulator tetratetracontane (TTC: $\mathrm{C}_{44} \mathrm{H}_{90}$ ). TTC was evaporated on $\mathrm{Cu}(100)$ with the substrate temperature of $300 \mathrm{~K}$ in a Knudsen cell. Real-time observation of crystallinity and orientation of the films was monitored by the reflection high-energy electron diffraction (RHEED).

Figure 1 shows the RHEED pattern for a 1 ML(mono-layer)thick TTC film grown on $\mathrm{Cu}(001)$. Clear diffraction patterns were observed when the incident electron beam parallel to the [110] azimuth of $\mathrm{Cu}(001)$, which indicates that a singlecrystalline TTC film grew heteroepitaxially on $\mathrm{Cu}(001)$ with its molecular long axis parallel to the [110] azimuth of $\mathrm{Cu}(001)$.
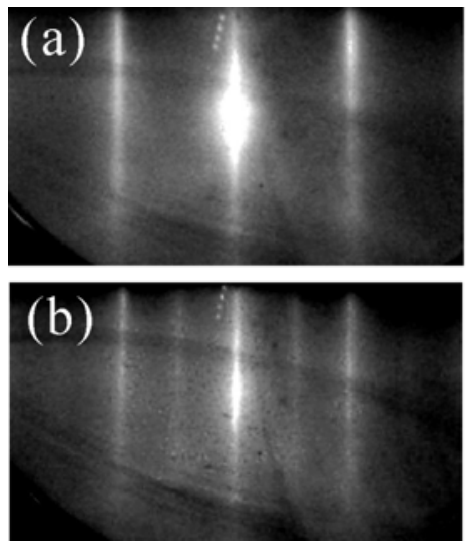

Figure 1. RHEED pattern for (a) the $\mathrm{Cu}$ substrate and (b) $1 \mathrm{ML}$ TTC grown on $\mathrm{Cu}(001)$. The incident electron beam is parallel to $\mathrm{Cu}[110]$. 
The streaks in the RHEED pattern show that the 1 ML film was atomically flat, i.e., the insulator/metal interface is indeed well-defined.

Figure 2 shows the polarization dependence of NEXAFS spectra for 1 ML-thick TTC/Cu(001) together with that for a 25 ML thick layer TTC. A pronounced pre-peak appears just below the bulk edge onset for the $1 \mathrm{ML}$ TTC. Since NEXAFS qualitatively provides information on the density of states of unoccupied states, the appearance of the pre-peak below the edge indicates that new states are formed below the vacuum level and above the Fermi level. Since the interaction between the organic molecule and the substrate is quite weak ( $\sim 10 \mathrm{~kJ} / \mathrm{mol} / \mathrm{CH}_{2}$ chain), which is in the physisorption rather than chemisorption regime, the pre-peak observed by NEXAFS should originate not from chemical bonds at the interface, but from the states formed by the proximity of the organic layer to a metal, i.e., metal-induced gap states in an organic material.

The pre-peak is greater for a grazing $\mathrm{x}$-ray incidence than for the normal incidence, indicating that the MIGS wave functions are oriented in the surface normal direction.

\section{AB-INITIO CALCULATION}

We have then performed a first-principles calculation based on the density functional theory with pseudopotentials and plane-wave basis. In the top panel of Figure 3 we show the atomic configuration considered in the actual calculation. The bottom panel of Fig.3 displays the local density of states at Fermi energy, where we can see an amplitude having significant magnitude at the interface and peaked at carbon sites. This result supports the formation of MIGS at the organic/metal interface. The MIGS wave functions are seen to be oriented in the surface normal direction, which agrees with the above experimental result.
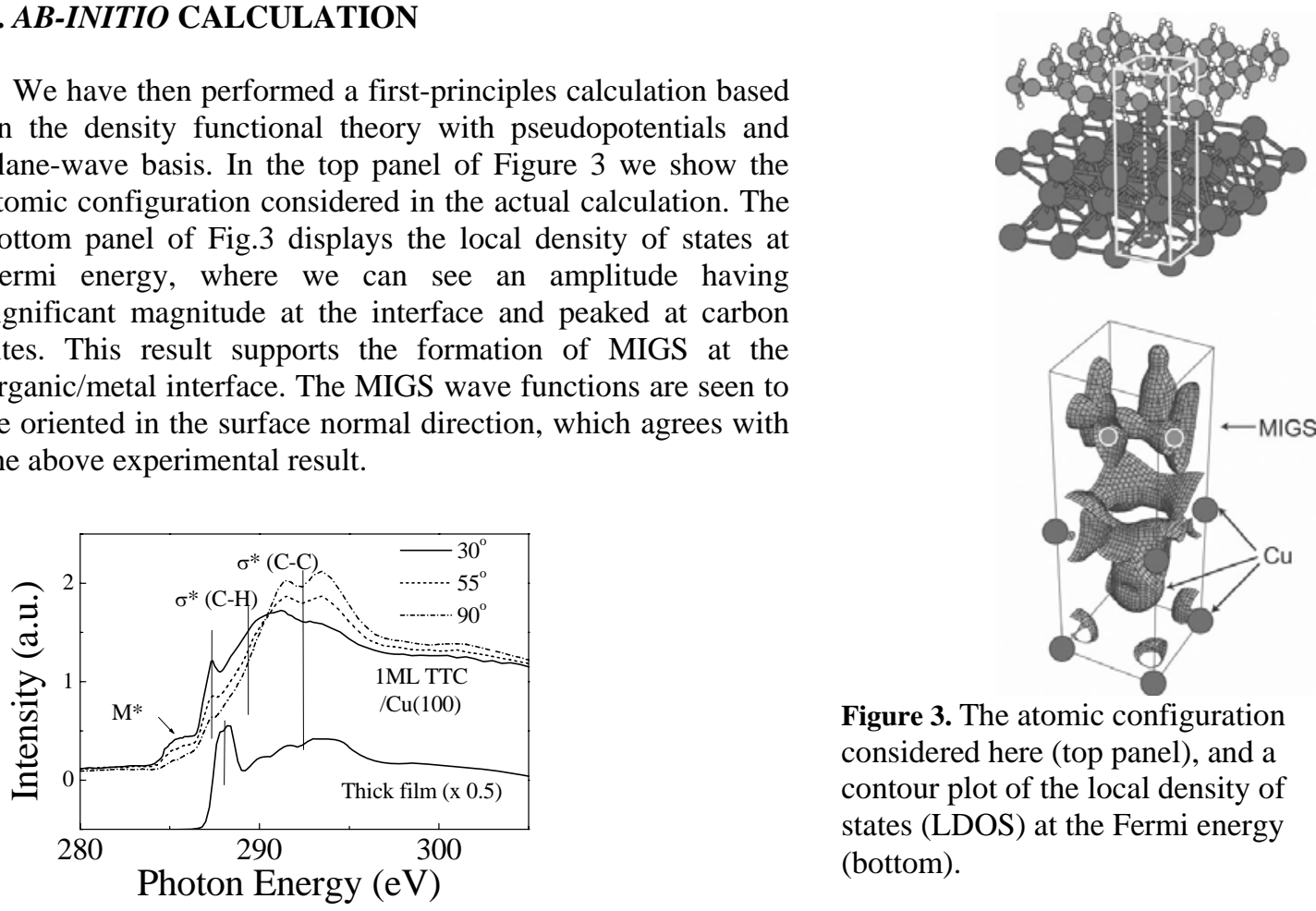

Figure 3. The atomic configuration considered here (top panel), and a contour plot of the local density of states (LDOS) at the Fermi energy (bottom).

Figure 2. The polarization dependence of C-K edge NEXAFS spectra for $1 \mathrm{ML}$ thick TTC/Cu(001) along with that for thick layer TTC for comparison. The x-ray incident angles are 90,55 and $30^{\circ}$. All the spectra are normalized by their edge-jump.

\section{Acknowledgments}

This work was supported by a creative scientific research project No. 14GS0207 from MEXT of Japan. One of the authors (M.K.) is also indebted to Foundation for C\&C Promotion for financial support.

\section{References}

[1] M. Kiguchi, R. Arita, G. Yoshikawa, Y. Tanida, M. Katayama, K. Saiki, A. Koma, and H. Aoki, Phys. Rev. Lett. 90 (2003) 196803.

[2] H. Kondo, F. Matsui, Y. Ehara, T. Yokoyama, and T. Ohta, Langmuir 17 (2001) 8178.

[3] Y. Hosoi, Y. Sakurai, M. Yamamoto, H. Ishii, Y. Ouchi, and K. Seki, Surf. Sci. 515 (2002) 157. 